\title{
Review
}

\section{Hypoxia and oxidative stress in breast cancer Oxidative stress: its effects on the growth, metastatic potential and response to therapy of breast cancer}

\author{
Nicholas S Brown and Roy Bicknell
}

\begin{abstract}
Molecular Angiogenesis Laboratory, Imperial Cancer Research Fund, Weatherall Institute of Molecular Medicine, John Radcliffe Hospital, University of Oxford, Oxford, UK
\end{abstract}

Correspondence: Dr Roy Bicknell, ICRF, WIMM, John Radcliffe Hospital, Oxford, OX3 9DS, UK. Tel: +44 1865 222457; fax: +44 1865 222431; e-mail: r.bicknell@icrf.icnet.uk

Received: 11 April 2001

Breast Cancer Res 2001, 3:323-327

Revisions requested: 31 May 2001

Revisions received: 19 June 2001

Accepted: 30 June 2001

(C) 2001 BioMed Central Ltd

Published: 23 July 2001

(Print ISSN 1465-5411; Online ISSN 1465-542X)

\begin{abstract}
Reactive oxygen species (ROS) damage DNA, but the role of ROS in breast carcinoma may not be limited to the mutagenic activity that drives carcinoma initiation and progression. Carcinoma cells in vitro and in vivo are frequently under persistent oxidative stress. In the present review, we outline potential causes of oxygen radical generation within carcinoma cells and explore the possible impact of oxidative stress on the clinical outcome of breast carcinoma.
\end{abstract}

Keywords: angiogenesis, apoptosis, breast cancer, metastasis, oxidative stress

\section{Introduction}

Oxygen radicals are continuously generated within mammalian cells, this being a consequence of the use of oxygen in aerobic respiration. Superoxide is generated within the mitochondria and is sequentially reduced to hydrogen peroxide and hydroxyl radicals. These species damage DNA, producing the mutations that initiate tumours and sustain progression [1]. Epidemiological studies suggest that a diet that is rich in antioxidants may help to prevent the development of breast carcinoma; this evidence contributed to recent UK Government advice that individuals should consume at least five portions of fruit or vegetables each day. The UK Department of Health has now translated this recommendation into initiatives such as the National School Fruit Scheme and the Five-aday Community Projects (www.doh.gov.uk). The role of ROS in breast carcinoma may not be limited to early mutagenic events, however. Carcinoma cells are frequently under persistent oxidative stress. Human tumour cell lines in vitro produce ROS at a far greater rate than do non- transformed cell lines [2], and markers of constitutive oxidative stress have been detected in samples from in vivo breast carcinomas [3,4]. 8-Hydroxy-2'-deoxyguanosine, one of the major oxidatively modified DNA base products, is almost 10 times more prevalent in invasive ductal breast carcinoma cells than in normal control samples from the same patient [3]. It appears unlikely that such a high level of oxidatively modified DNA is exclusively due to the mutagens that initiated the tumour. Persistent oxidative stress within carcinoma cells may instead be responsible for the accumulation of 8-hydroxy-2'-deoxyguanosine.

\section{Causes of carcinoma cell oxidative stress} Alterations to metabolic pathways in tumour cells Oxygen radicals are not only generated in the mitochondria. Neutrophils and macrophages produce ROS via a plasma membrane bound nicotinamide adenine dinucleotide phosphate, reduced form (NADPH)-oxidase. The radicals are generated for cell killing and bactericidal activities. The NADPH-oxidase is not exclusive to these cells,

$\mathrm{HIF}=$ hypoxia inducible factor; MAPK = mitogen-activated protein kinase; MMP = matrix metalloproteinase; NADPH = nicotinamide adenine dinucleotide phosphate, reduced form; NOS = nitric oxide synthase; ROS = reactive oxygen species; VEGF = vascular endothelial growth factor. 
however. A panel of human tumour cell lines were shown to produce large quantities of hydrogen peroxide in vitro [2]. The hydrogen peroxide production was prevented by diphenyleneiodonium, which is an inhibitor of the flavoprotein component of the NADPH-oxidase. Tumour cells may overproduce ROS because the NADPH-oxidase is regulated by the GTPase Rac1, which is itself downstream of the proto-oncogene Ras [5].

Carcinoma cell oxidative stress can also be induced by thymidine phosphorylase, an enzyme that is overexpressed in the majority of breast carcinomas. Thymidine phosphorylase catabolizes thymidine to thymine and 2-deoxy-Dribose-1-phosphate; the latter is a very powerful reducing sugar that rapidly glycates proteins, generating oxygen radicals within the carcinoma cell. Thymidine phosphorylase activity has been shown to induce carcinoma cell oxidative stress in vitro [6]. The frequent upregulation of thymidine phosphorylase in human breast tumours suggests that this may be an important cause of oxidative stress in breast cancer. Oxidative stress within breast carcinoma may also be caused by a breast specific mechanism, namely the metabolism of oestrogenic hormones by lactoperoxidase. Lactoperoxidase, an enzyme that is produced within the mammary gland, catalyzes the one-electron oxidation of $17 \beta$-oestradiol to a reactive phenoxyl radical [7].

The above metabolic changes will elicit carcinoma cell oxidative stress both in vitro and in vivo. We now consider further mechanisms by which tumour oxidative stress may arise. These, however, are dependent on the in vivo setting of the cancer.

\section{An inadequate tumour vascular network}

A breast tumour rapidly outgrows its blood supply, leading to glucose deprivation and hypoxia. Glucose deprivation rapidly induces cellular oxidative stress within the MCF-7 breast carcinoma cell line, although it does not cause oxidative stress in nontransformed cell lines $[8,9]$. This may be because glucose deprivation depletes intracellular pyruvate within the breast carcinoma cell, preventing the decomposition of endogenous oxygen radicals [8].

Breast carcinomas usually support their growth by stimulating blood vessel development (angiogenesis). Blood flow within these new vessels is often chaotic, causing periods of hypoxia followed by reperfusion. Reperfusion after myocardial infarction or cerebral ischaemia is known to cause the generation of ROS. Oxygen radical production during reperfusion may therefore be a cause of oxidative stress within breast carcinomas.

\section{Macrophage infiltration of the tumour}

Breast tumours are frequently infiltrated by large numbers of macrophages. These may contribute to carcinoma cell oxidative stress, as tumour-associated macrophages have been shown to deliver a sublethal oxidative stress to murine mammary tumour cells [10]. This may be due to oxygen radical production by the macrophages. In addition, tumour necrosis factor- $\alpha$ is secreted by tumourassociated macrophages, and is known to induce cellular oxidative stress [11].

\section{Therapeutic interventions}

Some anticancer therapies may add to the oxidative stress within breast carcinomas. The chemotherapeutic agents doxorubicin, mitomycin $\mathrm{C}$, etoposide and cisplatin are superoxide generating agents [12]. Radiotherapy and photodynamic therapy generate oxygen radicals within the carcinoma cell. The antioestrogen tamoxifen, increasingly used alongside other breast cancer therapies, has also been shown to induce oxidative stress within carcinoma cells in vitro [13].

\section{Consequences of carcinoma cell oxidative stress \\ Increased mutation rate and accelerated tumour progression}

As previously noted, oxygen radicals are powerful DNA damaging agents. ROS cause strand breaks, alterations in guanine and thymine bases, and sister chromatid exchanges [1]. This may inactivate additional tumour suppressor genes within tumour cells, or further increase expression of proto-oncogenes. Genetic instability due to persistent carcinoma cell oxidative stress will therefore increase the malignant potential of the tumour [2].

\section{Activation of growth-promoting signalling pathways}

Sublethal oxidative stress promotes cell proliferation in vitro, with both superoxide and hydrogen peroxide stimulating growth [14]. Proliferation in response to hydrogen peroxide may be due to the activation of mitogen-activated protein kinases (MAPKs). HeLa cells treated with hydrogen peroxide undergo a sustained activation of all three MAPK pathways [15]: extracellular signal related protein kinase; c-Jun amino-terminal kinase/stress-activated protein kinase; and p38. Hyperphosphorylation of c-Jun by oxidative stress activates activator protein-1 in MCF-7 breast carcinoma cells, a response that stimulates proliferation [16], and multidrug-resistant human breast carcinoma cells rapidly activate extracellular signal related protein kinase- 2 when stressed by glucose deprivation [9]. In addition, ROS may trigger mitosis via MAPK independent mechanisms. Oncogenic Ras causes ROS production by activating Rac1 and the NADPH-oxidase. In Ras-transformed human fibroblasts, ROS drive cell cycle progression without the activation of MAPK pathways [17].

\section{Adaptation to oxidative stress, resulting in increased resistance to therapy}

Severe oxidative stress leads to apoptosis. Conversely, persistent oxidative stress at sublethal levels may cause 
resistance to apoptosis. The induction of programmed cell death by ROS is dependent on p53 in both mouse and human cell lines [18]. Constitutive oxidative stress within breast carcinoma cells may therefore accelerate the selection of p53 knockout tumour cell clones, which have an apoptosis resistant phenotype. Persistent oxidative stress may also cause adaptive responses within the tumour cell that confer resistance to apoptosis. The antioxidant thiols thioredoxin and metallothionein are rapidly upregulated in response to oxidative stress $[12,19]$, and the antioxidants malondialdehyde, superoxide dismutase, glutathione peroxidase and catalase show increased expression or activity in breast tumour tissue as compared with normal controls [4]. An upregulation of anti-ROS defences in cancer cells may explain why tumour cell lines in vitro are extremely resistant to cytolysis by hydrogen peroxide [20]. In addition, antiapoptotic Akt (protein kinase B) is activated by hydrogen peroxide [21].

An antiapoptotic response to chronic oxidative stress may have severe implications for anticancer therapy. As outlined above, radiotherapy, photodynamic therapy and many chemotherapies generate oxygen radicals. Their antitumour activity is to a degree dependent on the induction of tumour cell apoptosis in response to oxidative stress and oxygen radical induced DNA damage [12]. Persistent oxidative stress within carcinoma cells may therefore cause resistance to therapy. Oxygen radicals might also increase drug resistance by increasing carcinoma cell expression of P-glycoprotein, the multidrug-resistance efflux pump [22].

\section{Increased blood supply to tumour cells}

Oxygen radicals increase tumour cell production of the angiogenic factors IL-8 and vascular endothelial growth factor (VEGF) [6]. Tumour cell oxidative stress also promotes secretion of the matrix metalloproteinase-1 (MMP-1), a collagenase that aids vessel growth within the tumour microenvironment [6]. Oxidative stress can therefore cause angiogenesis within breast carcinoma. Hypoxia and oxidative stress may be found together within the tumour, and VEGF production within oxidatively stressed breast carcinomas may be augmented by synergy between oxygen radicals and tumour hypoxia. Levels of the hypoxia inducible factor-1 (HIF-1) may be increased by oxygen radicals $[23,24]$, implying that oxidatively stressed carcinoma cells might show increased HIF-1 induction during hypoxia and therefore produce more VEGF (Fig. 1).

Oxidative stress may also increase the blood supply to breast carcinoma by triggering vasodilatation. Hydrogen peroxide induces inducible nitric oxide synthase (NOS) in cytokine stimulated rat pleural mesothelial cells [25], raising the possibility that oxidatively stressed breast tumour cells might show increased expression of inducible NOS. The nitric oxide produced would activate cGMP within nearby smooth muscle cells, leading to vasodilata-
Figure 1

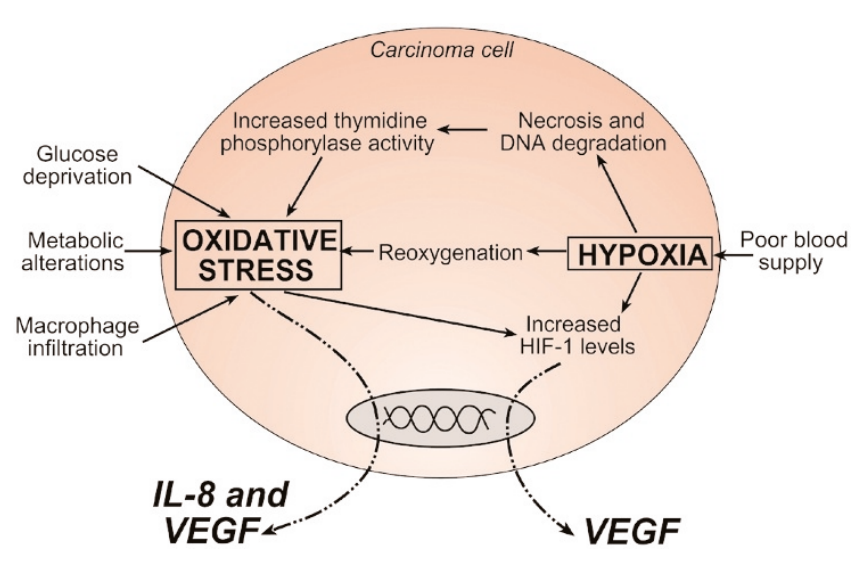

Oxygen radicals and oxygen insufficiency (hypoxia) co-operatively promote tumour angiogenesis. Breast carcinomas frequently outgrow their blood supply, leading to oxygen insufficiency (hypoxia) within the tumour. Hypoxia causes necrosis, and DNA is subsequently degraded to its constituent bases. Any thymidine released is catabolized by thymidine phosphorylase, an enzyme that is frequently overexpressed within breast carcinomas. Thymidine phosphorylase activity causes oxygen radical production, as described by Brown et al [6].

Reoxygenation of the tumour after hypoxia will drive additional oxygen radical formation. Breast tumours are also oxidatively stressed by nonhypoxic mechanisms, such as glucose deprivation, metabolic alterations and macrophage infiltration. Hypoxia causes the accumulation of the transcription factor HIF-1, which promotes transcription of the angiogenic factor VEGF. HIF-1 levels may also be increased by oxygen radicals. In addition, oxygen radicals increase production of the angiogenic factors VEGF and IL-8 via HIF-1independent mechanisms.

tion. Vasodilatation could also be triggered by carbon monoxide, because oxidative stress powerfully induces heme oxygenase-1 [6], which degrades heme to biliverdin and carbon monoxide. Carbon monoxide, like nitric oxide, activates cGMP.

\section{Increased risk of metastasis}

Blood vessel growth within the breast tumour microenvironment increases the risk of blood-borne metastasis. Angiogenesis may also promote lymphatic dissemination, a common occurrence in breast carcinoma, by elevating tumour interstitial pressure. These are not the only mechanisms by which oxidative stress can aid tumour spread, however. Oxygen radicals may also augment tumour cell migration, increasing the risk of invasion and metastasis. The p38 MAPK is activated by oxidative stress [15], and the phosphorylation of heat shock protein-27 by $\mathrm{p} 38$ MAPK has been shown to induce changes in actin dynamics [26]. Phosphorylated heat shock protein-27 promotes the migration of MDA-MB-231 breast cancer cells on laminin-5 in vitro [27]. As noted earlier, Rac1 can activate the NADPH-oxidase in tumour cells, causing superoxide production. ROS have been shown to mediate the role of Rac1 in actin cytoskeleton reorganization [28]. 
Oxidative stress within breast tumours may also facilitate invasion and metastasis by activating MMPs and inhibiting antiproteases. MMP-2 is a gelatinase that is believed to play a major role in breast cancer invasion and metastasis. High levels of MMP-2 correlate with poor prognosis in breast cancer patients [29] and active MMP-2 is detected more frequently in malignant than in benign breast tumours. ROS have been shown to activate MMP-2, possibly by the reaction of oxygen radicals with thiol groups within MMP-2 [30]. Protease inhibitors, such as $\alpha_{1}$-proteinase inhibitor and plasminogen activator inhibitor, may be inactivated by oxidation of methionine residues at their active sites [31]. This facilitates the activity of various proteases, increasing invasion and the likelihood of metastasis. For example, plasminogen activator is believed to play a role in metastasis [2].

Murine mammary carcinoma cells that are exposed to sublethal oxidative stress exhibit decreased attachment to immobilized laminin and fibronectin [10]. Reduced tumour cell adhesion to basement membrane components increases the probability that the cells will detach and enter the blood vessels or lymphatic system. In addition, treatment of these cells with hydrogen peroxide, before intravenous injection into mice, enhanced lung metastasis formation. This implies that oxidative stress may aid the seeding of metastatic tumour cells [10]. Finally, ROS within the tumour microenvironment may promote metastasis by increasing vascular permeability, either by direct damage to endothelial cells or by the upregulation of inducible NOS and heme oxygenase- 1 previously proposed.

\section{Conclusion}

Carcinoma cells synthesize ROS at an elevated rate in vitro, and many tumours in vivo appear to be under persistent oxidative stress. The present review outlines potential causes of carcinoma cell oxidative stress in vitro and in vivo, and summarizes mechanisms by which oxygen radicals may affect the outcome of breast cancer. Future experiments will reveal the extent to which oxidative stress influences the prognosis of breast carcinoma. If oxygen radicals do lead to a poorer prognosis, then antioxidants may be of therapeutic value. This is an exciting possibility, because antioxidants are drugs of low toxicity. Evidence from other tumour types suggests that antioxidants may indeed be of use against breast cancer. For example, transfection of human melanoma cells with cDNA encoding the antioxidant enzyme manganese superoxide dismutase suppressed their malignant phenotype. The cells lost their ability to form colonies on soft agar, and no longer formed tumours in nude mice [32].

\section{References}

1. Wiseman $H$, Halliwell $B$ : Damage to DNA by reactive oxygen and nitrogen species: role in inflammatory disease and progression to cancer. Biochem J 1996, 313:17-29.
2. Szatrowski TP, Nathan CF: Production of large amounts of hydrogen peroxide by human tumor cells. Cancer Res 1991, 51:794-798.

3. Toyokuni S, Okamoto K, Yodoi J, Hiai H: Persistent oxidative stress in cancer. FEBS Lett 1995, 358:1-3.

4. Portakal O, Ozkaya O, Erden Inal M, Bozan B, Kosan M, Sayek I: Coenzyme Q10 concentrations and antioxidant status in tissues of breast cancer patients. Clin Biochem 2000, 33:279284.

5. Sundaresan M, Yu Z-X, Ferrans VJ, Sulciner DJ, Gutkind JS, Irani K, Goldschmidt-Clermont PJ, Finkel T: Regulation of reactiveoxygen-species generation in fibroblasts by Rac1. Biochem $J$ 318:379-382.

6. Brown NS, Jones A, Fujiyama C, Harris AL, Bicknell R: Thymidine phosphorylase induces carcinoma cell oxidative stress and promotes secretion of angiogenic factors. Cancer Res 2000, 60:6298-6302.

7. Sipe HJ Jr, Jordan SJ, Hanna PM, Mason RP: The metabolism of 17 beta-estradiol by lactoperoxidase: a possible source of oxidative stress in breast cancer. Carcinogenesis 1994, 15: 2637-2643.

8. Spitz DR, Sim JE, Ridnour LA, Galoforo SS, Lee YJ: Glucose deprivation-induced oxidative stress in human tumor cells. A fundamental defect in metabolism? Ann N Y Acad Sci 2000, 899:349-362.

9. Lee YJ, Galoforo SS, Berns CM, Chen JC, Davis BH, Sim JE, Corry PM, Spitz DR: Glucose deprivation-induced cytotoxicity and alterations in mitogen-activated protein kinase activation are mediated by oxidative stress in multidrug-resistant human breast carcinoma cells. J Biol Chem 1998, 273:52945299.

10. Kundu N, Zhang S, Fulton AM: Sublethal oxidative stress inhibits tumor cell adhesion and enhances experimental metastasis of murine mammary carcinoma. Clin Exp Metastasis 1995, 13:16-22.

11. Fulton AM, Chong YC: The role of macrophage-derived TNF $\alpha$ in the induction of sublethal tumor cell DNA damage. Carcinogenesis 1992, 13:77-81.

12. Yokomizo $A$, Ono $M$, Nanri $H$, Makino $Y$, Ohga $T$, Wada $M$, Okamoto T, Yodoi J, Kuwano M, Kohno K: Cellular levels of thioredoxin associated with drug sensitivity to cisplatin, mitomycin C, doxorubicin, and etoposide. Cancer Res 1995, 55: 4293-4296.

13. Ferlini C, Scambia G, Marone M, Distefano M, Gaggini C, Ferrandina $G$, Fattorossi A, Isola G, Benedetti Panici P, Mancuso S: Tamoxifen induces oxidative stress and apoptosis in oestrogen receptor-negative human cancer cell lines. $\mathrm{Br} J$ Cancer 1999, 79:257-263.

14. Burdon RH: Superoxide and hydrogen peroxide in relation to mammalian cell proliferation. Free Radic Biol Med 1995, 18: 775-794.

15. Wang X, Martindale JL, Liu Y, Holbrook NJ: The cellular response to oxidative stress: influences of mitogen-activated protein kinase signalling pathways on cell survival. Biochem J 1998, 333:291-300.

16. Schiff R, Reddy P, Ahotupa M, Coronado-Heinsohn E, Grim M, Hilsenbeck SG, Lawrence R, Deneke S, Herrera R, Chamness GC, Fuqua SA, Brown PH, Osborne CK: Oxidative stress and AP-1 activity in tamoxifen-resistant breast tumors in vivo. $J$ Natl Cancer Inst 2000, 92:1926-1934.

17. Irani K, Xia Y, Zweier JL, Sollott SJ, Der CJ, Fearon ER, Sundaresan M, Finkel T, Goldschmidt-Clermont PJ: Mitogenic signaling mediated by oxidants in Ras-transformed fibroblasts. Science 1997, 275:1649-1652.

18. Yin Y, Solomon G, Deng C, Barrett JC: Differential regulation of $\mathrm{p} 21$ by $\mathrm{p} 53$ and $\mathrm{Rb}$ in cellular response to oxidative stress. Mol Carcinog 1999, 24:15-24.

19. Lazo JS, Kuo SM, Woo ES, Pitt BR: The protein thiol metallothionein as an antioxidant and protectant against antineoplastic drugs. Chem Biol Interact 1998, 111-112:255-262.

20. O'Donnell-Tormey J, DeBoer CJ, Nathan CF: Resistance of human tumor cells in vitro to oxidative cytolysis. J Clin Invest 1985, 76:80-86.

21. Shaw $M$, Cohen $P$, Alessi DR: The activation of protein kinase $B$ by $\mathrm{H}_{2} \mathrm{O}_{2}$ or heat shock is mediated by phosphoinositide 3kinase and not by mitogen-activated protein kinase-activated protein kinase-2. Biochem J 1998, 336:241-246. 
22. Ziemann C, Burkle A, Kahl GF, Hirsch-Ernst Kl: Reactive oxygen species participate in mdr1b mRNA and P-glycoprotein overexpression in primary rat hepatocyte cultures. Carcinogenesis 1999, 20:407-414.

23. Chandel NS, McClintock DS, Feliciano CE, Wood TM, Melendez JA, Rodriguez AM, Schumacker PT: Reactive oxygen species generated at mitochondrial complex III stabilize hypoxiainducible factor- $1 \alpha$ during hypoxia: a mechanism of $\mathrm{O}_{2}$ sensing. J Biol Chem 2000, 275:25130-25138.

24. Richard DE, Berra E, Pouyssegur J: Nonhypoxic pathway mediates the induction of hypoxia-inducible factor $1 \alpha$ in vascular smooth muscle cells. J Biol Chem 2000, 275:26765-26771.

25. Milligan SA, Owens MW, Grisham MB: Augmentation of cytokine-induced nitric oxide synthesis by hydrogen peroxide. Am J Physiol 1996, 271:L114-L120.

26. Huot J, Houle F, Marceau F, Landry J: Oxidative stress-induced actin reorganization mediated by the p38 mitogen-activated protein kinase/heat shock protein 27 pathway in vascular endothelial cells. Circ Res 1997, 80:383-392.

27. Rust W, Kingsley K, Petnicki T, Padmanabhan S, Carper SW, Plopper GE: Heat shock protein 27 plays two distinct roles in controlling human breast cancer cell migration on laminin-5. Mol Cell Biol Res Commun 1999, 1:196-202.

28. Moldovan L, Irani K, Moldovan NI, Finkel T, Goldschmidt-Clermont PJ: The actin cytoskeleton reorganization induced by Rac1 requires the production of superoxide. Antiox Redox Signal 1999, 1:29-43.

29. Duffy MJ, Maguire TM, Hill A, McDermott E, O'Higgins N: Metalloproteinases: role in breast carcinogenesis, invasion and metastasis. Breast Cancer Res 2000, 2:252-257.

30. Rajagopalan S, Meng XP, Ramasamy S, Harrison DG, Galis ZS: Reactive oxygen species produced by macrophage-derived foam cells regulate the activity of vascular matrix metalloproteinases in vitro. Implications for atherosclerotic plaque stability. J Clin Invest 1996, 98:2572-2579.

31. Swaim MW, Pizzo SV: Methionine sulfoxide and the oxidative regulation of plasma proteinase inhibitors. J Leukoc Biol 1988, 43:365-379.

32. Church SL, Grant JW, Ridnour LA, Oberley LW, Swanson PE, Meltzer PS, Trent JM: Increased manganese superoxide dismutase expression suppresses the malignant phenotype of human melanoma cells. Proc Natl Acad Sci USA 1993, 90: 3113-3117. 